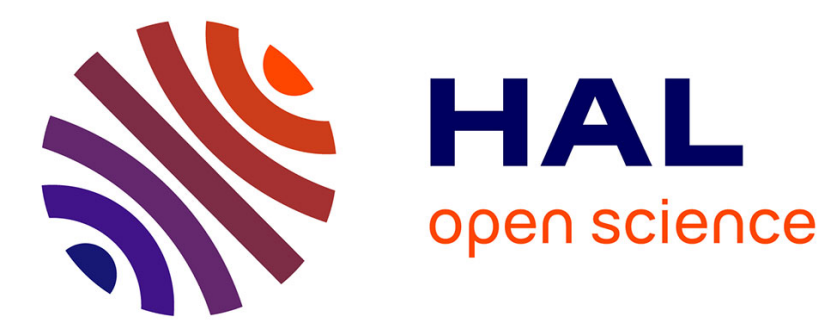

\title{
Contribution to the generation of tool paths in a cam system
}

Arnaud Larue, Bernard Anselmetti

\section{To cite this version:}

Arnaud Larue, Bernard Anselmetti. Contribution to the generation of tool paths in a cam system. 4th international conference on integrated design and manufacturing in mechanical engineering, 2002, Clermont-Ferrand, France. 10.1007/978-94-017-0161-7_22 . hal-00098374

\section{HAL Id: hal-00098374 https://hal.science/hal-00098374}

Submitted on 25 Feb 2018

HAL is a multi-disciplinary open access archive for the deposit and dissemination of scientific research documents, whether they are published or not. The documents may come from teaching and research institutions in France or abroad, or from public or private research centers.
L'archive ouverte pluridisciplinaire HAL, est destinée au dépôt et à la diffusion de documents scientifiques de niveau recherche, publiés ou non, émanant des établissements d'enseignement et de recherche français ou étrangers, des laboratoires publics ou privés. 


\title{
CONTRIBUTION TO THE GENERATION OF TOOL PATHS IN A CAM SYSTEM
}

\author{
A. Larue and B. Anselmetti \\ LURPA, ENS de Cachan, France
}

Abstract: The flank milling of complex forms is a very effective process from the point of view of productivity and surface quality. Many works deal with research on the optimal positioning of the tool which is considered as a rigid body in order to minimize tool path errors. The purpose of our work is to integrate the compensation of the tool distortions in this optimal positioning calculation. In flank milling with long tools, the distortion of the cutter generates a significant wave (that can reach $0,6 \mathrm{~mm}$ ) on the machined surface due to the effects of the helical angle and the radial force which varies during the cutter rotation. After detailing an analysis of the force evolution and the associated model calculation, we will present a test protocol, that can be implemented in industry, in order to characterize the model parameters as a function of the couple tool-workpiece material. Then we will present a test to assess our prediction model of the straightness defects of the machined surface according to all machining parameters. These results make it possible to make up for defects by applying a translation to the tool in 3 -axis and by applying a translation combined with a rotation in 5-axis milling.

Key words: Identification, Force model, Compensation, Tool paths

\section{INTRODUCTION}

The flank machining of complex forms is currently a strong industrial issue. The objective is to machine a high quality surface while minimizing manufacturing times. A lot of the research deals with the generation of an 
optimal positioning of the tool which is considered as a rigid body in order to minimize tool paths errors [1], [2], [3]. These approaches ignore the cutting process's intrinsic elements, in particular the tool distortions which can be significant (about $0.6 \mathrm{~mm}$ for a $20 \mathrm{~mm}$ diameter long cutter and a raised material flow in medium-hard steel). Our objective is to take into account the tool deformations in tool path calculations for flank milling of free forms. This article qualitatively analyses the flank milling process and proposes a method to assess the cutting forces and the tool distortions. A test protocol, that can be implemented in industry is proposed to characterize the various model parameters as a function of a given couple tool-workpiece material. The defects are then compensated by a 3 axis tool translation.

\section{ANALYSIS OF THE CUTTING FORCE}

In order to highlight the tool distortion, a plane was machined with a high speed steel cutter of $20 \mathrm{~mm}$ diameter and $90 \mathrm{~mm}$ of length, with a progressive radial engagement from 0.5 to $3 \mathrm{~mm}$ and a constant feedrate per tooth $\mathrm{fz}=0.2 \mathrm{~mm} / \mathrm{rev}$. For example, for a radial engagement of $3 \mathrm{~mm}$, the distortion generates a variation of localization of $0.6 \mathrm{~mm}$ with a wave giving a variation of straightness of $0.2 \mathrm{~mm}$ (figure 1).

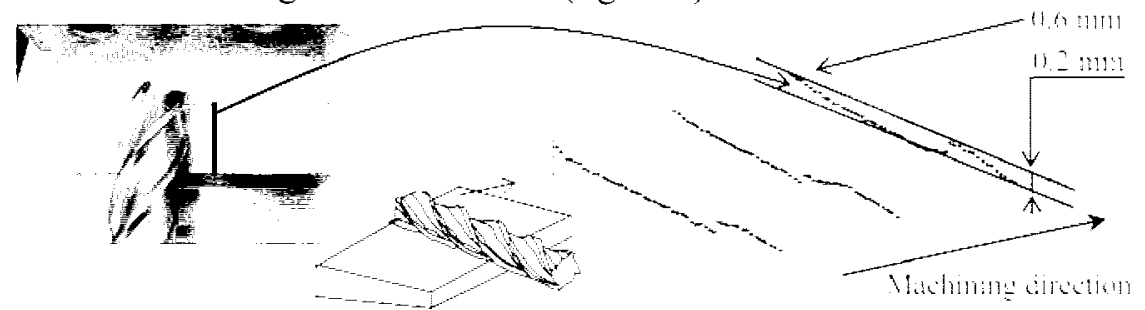

Figure -1. Evaluation of the machining deviation amplitude.

To model the tool distortion, it is necessary to understand the evolution of the cutting force. Figure 2 shows the cutting process :
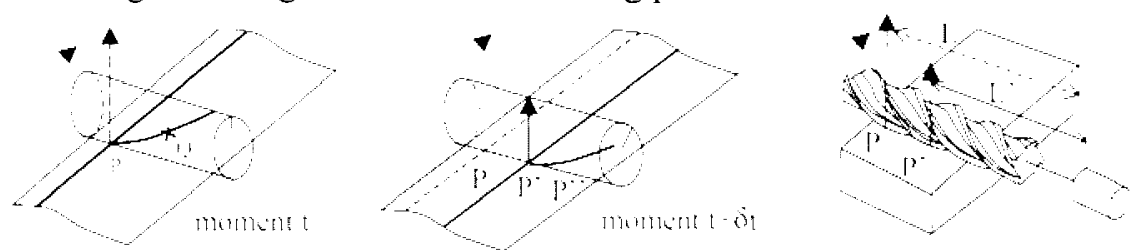

Figure-2. Displacement of the generating point.

The cutting edge of the milling tool is a helix. The surface is thus obtained by a generating point which moves along the milling tool generatrix during the cutter rotation. At moment $\mathrm{t}$, the generating point is at $\mathrm{P}$ at a distance $\mathrm{L}$ from the housing. At moment $\mathrm{t}+\delta \mathrm{t}$, the point is at $\mathrm{P}^{\prime}$ at a distance 
L'. An elementary cutting edge placed at point $Q$ which is inside the material (figure 3) cuts an elementary chip section which depends on the engagement angle at this point. An elementary cutting pressure is thus applied at this point. This force generates an elementary inflection of the cutter. The distortion at point $\mathrm{P}$ is thus the sum of the elementary distortions for the whole length in mesh of the cutting edges simultaneously engaged in the workpiece material.

The contact surface between the cutter and the workpiece material was developed in figure 3 which represents the cutter in three angular positions. The evolution of the chip section along the cutting edge, and the normal density of the force for each point $Q$ are identified according to the angular position $\beta$ at this point. $\alpha$ is the total engagement angle of the cutter in the workpiece material.

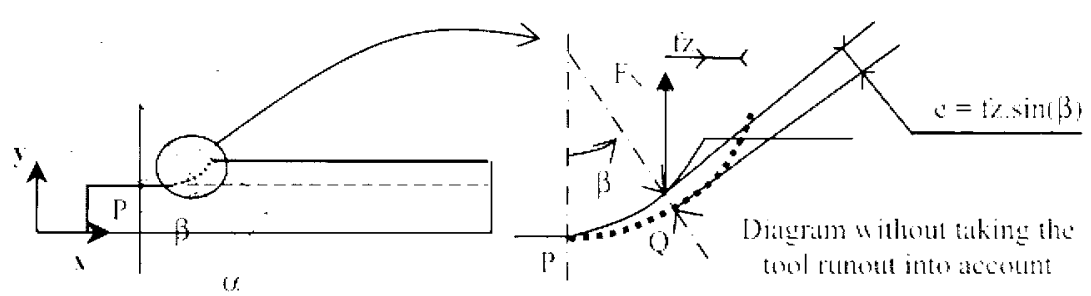

Evolution of:

Somal tore without taking the tont runout into atcount (hip section - - - -
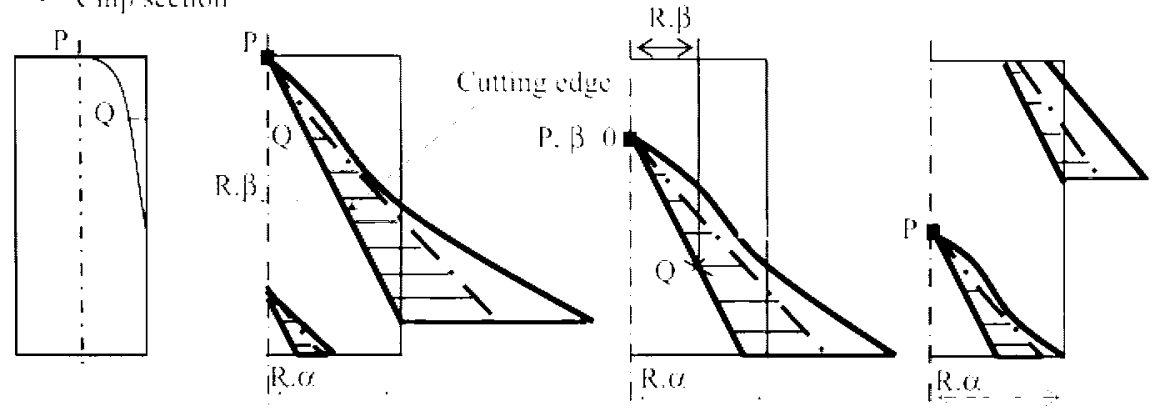

Figure -3. Radial Force evolution on helical developped profile.

During rotation, distortion is variable, in particular because of the distances from the generating point $P$ and points $Q$ to the housing variation. Moreover, there can be one or 2 teeth completely engaged. This variation of distortion as a function of point $\mathrm{P}$ sometimes creates a significant wave in a machined surface section. 


\section{DISTORTION MODEL OF THE MILLING TOOL}

There are numerous works concerning the static force model study [4], [5], [6]. Our modeling of the forces makes use of the basic principles of the Kline and DeVor model [7], [5], [8]. This approach allows for the prediction of the cutting force and is based on a static equilibrium of the tool fitted in the spindle cone.

\subsection{Influence of the various factors on distortion}

We suppose that the defects observed are mainly due to the cutter distortion. This assumption was validated by checking. The machined parts are rather massive and firmly clamped to be considered as a rigid body. The fixture is also very rigid (vice or table fixture). The spindle was subjected to a static force equivalent to the machining force [8] : the distance variation between the spindle and the fixture $(\sim 0.01 \mathrm{~mm})$ is is very low in relation to the observed defects. Similarly, the static distortion of the tool holder is also negligible. The distortions of components other than the milling tool will thus be ignored. The cutter will be considered as a beam fixed in the milling tool holder. A test also showed that the flexion was not deviated by the helical shape of the cutting edges.

\subsection{Modeling of the distortions}

The method that we propose differs from the Kline and DeVor approach as re-envisaged by Seo [6] : Our calculation estimates the generating point of the cutting edge distortion by adding up the distortions due to the elementary forces distributed along the cutting edge.

The model illustrated by figure 4 determines the range of deformation due to normal forces on the machined surface for each elementary length of the cutting edges in mesh.

The cutting pressure is assumed to follow the model (eq.(1); eq.(2)) :

$$
\begin{aligned}
& \Rightarrow \text { Radial force }: \mathrm{Kr}=\mathrm{Kr}_{\mathrm{o}} \cdot \mathrm{ep}^{-0.3}(\mathrm{ep}=\text { depth of cut }) \\
& \Rightarrow \text { Tangential force }: \mathrm{Kt}=\mathrm{Kt}_{0} \cdot \mathrm{ep}^{-0.3}
\end{aligned}
$$

Coefficients $\mathrm{Kr}_{\mathrm{o}}$ and $\mathrm{Kt}_{\mathrm{o}}$ depend in particular on the tool angles and the machined material. The exponent -0.3 is a term which is considered as constant for a wide material range. After the tool is broken down into elementary discs whose thickness is ea, the two components $\mathrm{dFt}$ and $\mathrm{dFr}$ of 
the elementary force can be calculated according to the angular position $\beta$ at point $Q$ considered (eq. (3) - eq. (4)).

$$
\begin{aligned}
& \Rightarrow \text { Radial force }: \mathrm{dFr}=\mathrm{Kr} \text {.ep.ea }=\mathrm{Kr}_{0}(\mathrm{fz} \cdot \sin (\beta))^{1-0.3} \text {.ea } \\
& \Rightarrow \text { Tangential force }: \mathrm{dFt}=\mathrm{Kt} \text {.ep.ea }=\mathrm{Kt}_{\mathrm{o}} .(\mathrm{fz} \cdot \sin (\beta))^{1-0.3} \text {.ea }
\end{aligned}
$$

The normal component to the machined surface $\mathrm{dN}$ can be calculated :

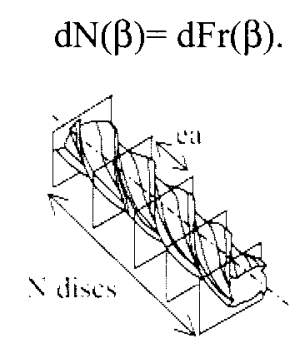

$$
\cos (\beta)+\mathrm{dFt}(\beta) \cdot \sin (\beta)
$$
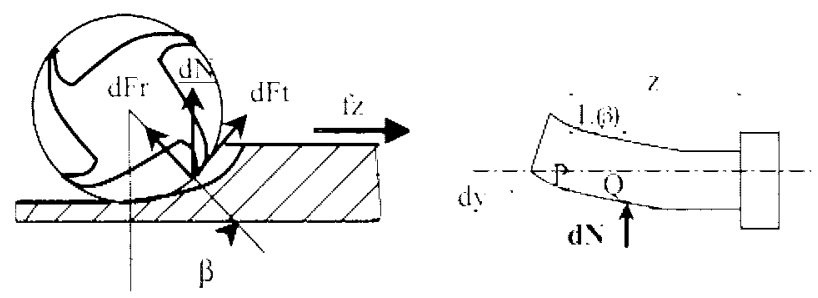

Figure -4. Details of the model used.

The distortion dy at point $\mathrm{P}$ due to force $\mathrm{dN}$ at point $\mathrm{Q}$ can be calculated by considering that the tool is a fixed beam of constant Igy moment of inertia :

$$
d y=\frac{1}{E \cdot \operatorname{Ig} y} \cdot d N(\beta) \cdot\left[\left(\frac{(z-L(\beta))^{3}}{3}\right) \cdot\left(\frac{(z-L(\beta))^{2} \cdot L(\beta)}{2}\right)\right]
$$

The radial engagement being small, there is only one tooth in mesh on each disc. At each point $P$, the theoretical $y_{\text {th }}$ is the sum of dy generated by all points $\mathrm{Q}$ of the cutting edges in mesh [8].

\section{COUPLE TOOL-WORKPIECE CHARACTERIZATION}

\subsection{Description of the test protocol}

The force model requires the identification of $\mathrm{Kr}_{0}, \mathrm{Kt}_{0}$, Igy et $\mathrm{E}$. The term E.Igy is identified thanks to a static test of the milling tool using a dynamometer [8].

The test is based on the machining of a plane. The part is measured on a three-dimensional coordinate measuring machine. A simulation model developed under Matlab software calculates the tool distortions for each 
point $\mathrm{P}$ on the surface by adjusting the parameters $\mathrm{Kr}_{0}, \mathrm{Kt}_{0}$ to reproduce the measured form as accurately as possible.

The test thus consists in flank machining a plane starting from a tilted raw plane of a known value (figure 5) giving a radial engagement ranging from 0.5 to $3 \mathrm{~mm}$. The milling tool engagement angle varies throughout the facing. On the two raw part sides, the two zones for which the radial depth of cut is $0.3 \mathrm{~mm}$ make it possible to easily readjust the measured dots cloud. The pure flank milling without end-milling is obtained by a groove in which the tool tip emerges. The calibration stage of the force model coefficients is then not disturbed by a parasitic friction of the tool point during the machining.

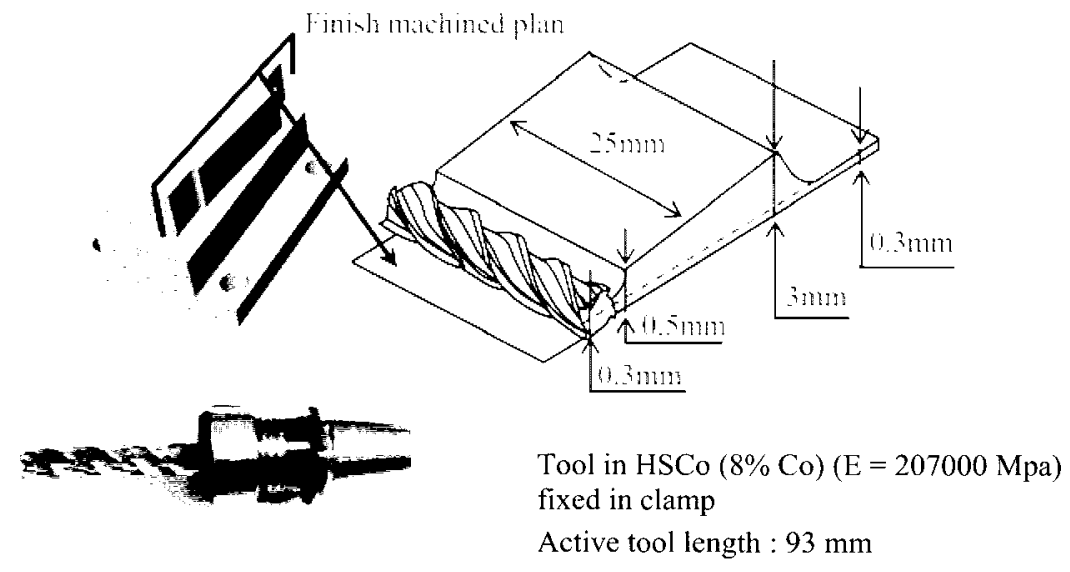

Figure -5. Test of tool - workpiece characterization.

Measurement is carried out in a rough-planing reference - mark built on the surfaces made with the tool to test and of the cutting conditions which must give negligible distortions of the tool (figure 5).

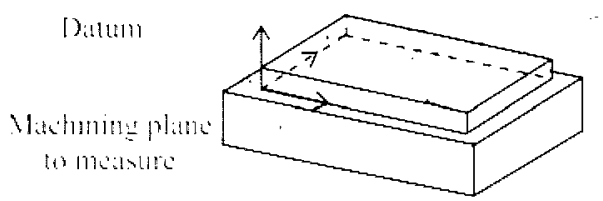

Thres planes machined by the tested tent with it small deptrat ont

Figure -6. Rough-planing reference - datum for the machined surface measurement.

The measurement, after processing in the calculation model, gives the difference between the points on the machined surface and the theoretical surface built relative to the reference-mark of rough-planing (figure 6). The model coefficients are obtained by minimizing the sum of the squared differences between the measured dot cloud and the simulated dot cloud [8]. Figure 7 a represents the simulated dots cloud (deflection ranging from 0 to $0.7 \mathrm{~mm}$ ). Figure $7 \mathrm{~b}$ shows the distribution of the differences between the 
points measured and the points simulated $( \pm 0.06 \mathrm{~mm})$. The most significant variations are on the part zone where radial engagement is largest.
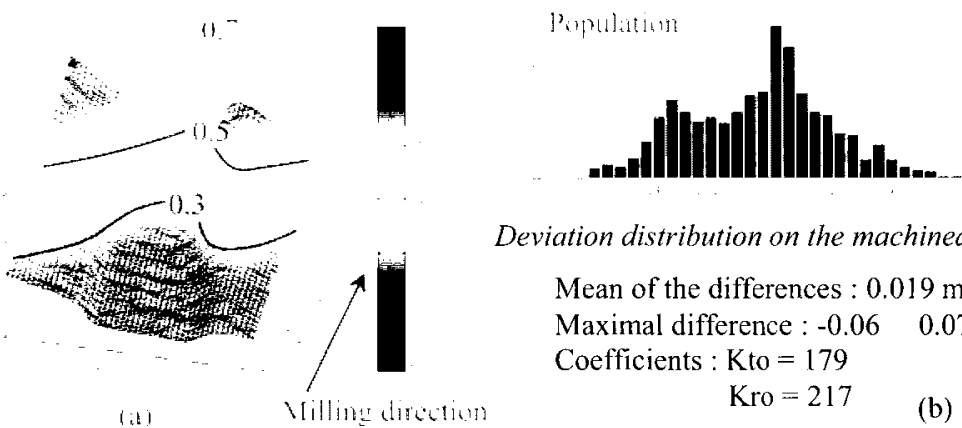

Deviation distribution on the machined surface

$$
\begin{aligned}
& \text { Mean of the differences : } 0.019 \mathrm{~mm} \\
& \text { Maximal difference : }-0.06 \quad 0.07 \mathrm{~mm} \\
& \text { Coefficients : Kto }=179 \\
& \text { Kro }=217
\end{aligned}
$$

Figure -7 . Numerical results for the HSS tool (tool diameter : $20 \mathrm{~mm}$, tool length : $94 \mathrm{~mm}$ ).

Figure 8 shows the results obtained with a shorter carbide tool.
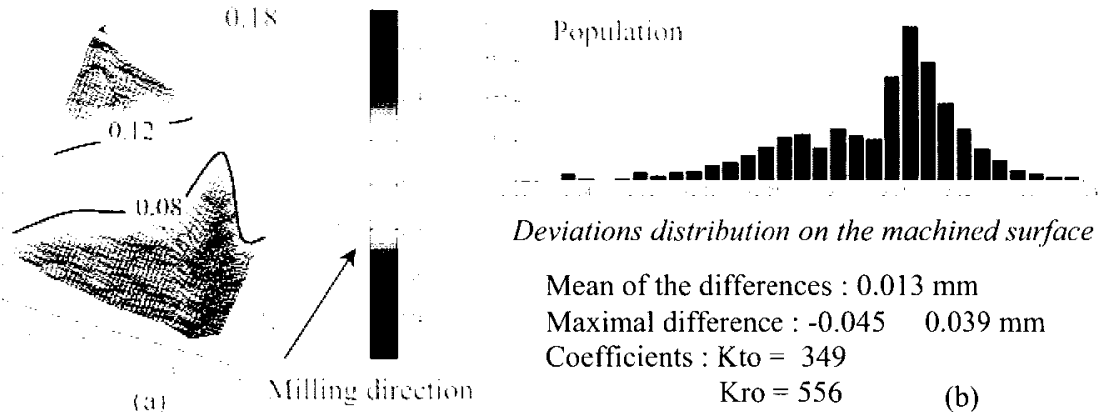

Figure -8 . Numerical results for the carbide tool (tool diameter : $20 \mathrm{~mm}$, tool length : $54 \mathrm{~mm}$ ).

This test protocol allows for the estimation of the force model coefficients and for the prediction of the machining defects with a sufficient precision next to the amplitude of the measured distortions. Figure 8 shows that most of the variations for the robust carbide tool are included in an interval of $\pm 0.03 \mathrm{~mm}$, which is acceptable as regards our precision target.

\subsection{Validation procedure of the prediction defect}

The test consists in realising a circular interpolation. In this case, for a given radial engagement, the engagement angle is smaller than for the plane. The measurement consists in identifying several generators between the altitudes $\mathrm{z}=0$ and $\mathrm{z}=30$.

The tests were performed for the two tools. The curves in figures 9 and 10 show the quality obtained by this simulation. 


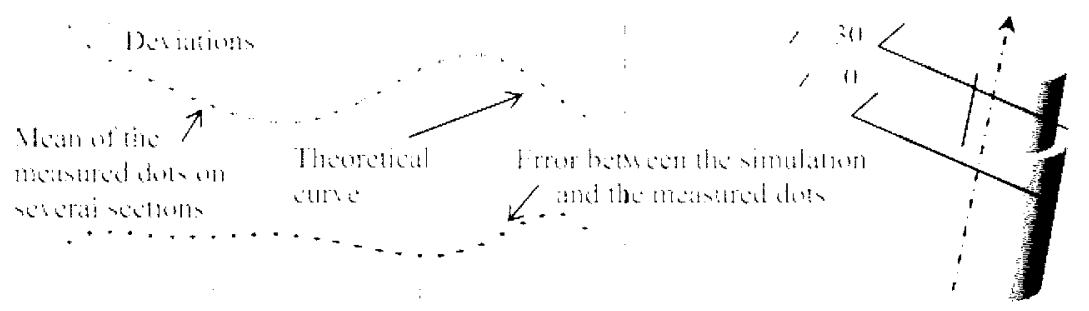

Figure -9. Validation of the identification procedure for a high speed steel tool.

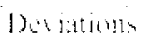

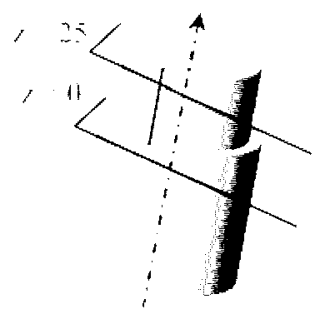

Figure -10. Validation of the identification procedure for a carbide tool.

The difference between the dots measured and the dots simulated is included in an interval of $\pm 0.02 \mathrm{~mm}$ over the first 20 millimetres. Considering the initial objectives, our modeling is validated. The model must however still be refined, because the top cylinder is still rather badly evaluated (approximately $0.05 \mathrm{~mm}$ deviation).

\section{COMPENSATION OF PREDICTED DEFECTS}

The mirror technique [6], [9] allows to partially make up for the defects generated by the working tool deflection. In order to achieve this, in each section, an average value of the distortion was calculated to generate a tool translation following the normal to the surface. The milling test of a plane starting from the rough surface used for characterization of the force model coefficients was realised by calculating the compensated tool path as shown in figure 11. After computation of the mean deformation to compensate in each section, the defects are interpolated with a non uniform cubic Bspline curve, which is then discretized to generate toolpath. The test results are illustrated in figure 12 .

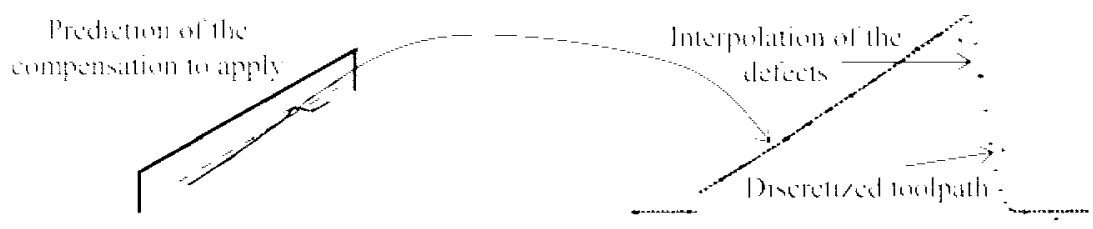

Figure-11. Generation of the compensated tool path. 


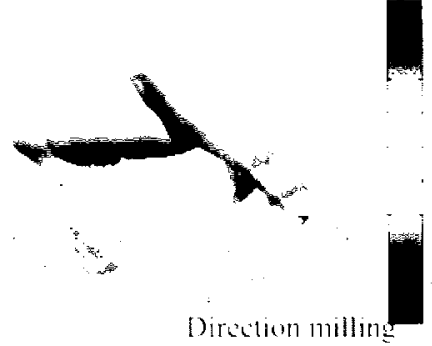

Direction millins

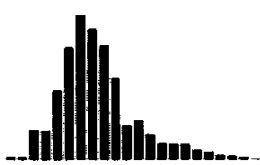

Difference e to $95 \%$ (with compensation) : $0.019 \mathrm{~mm}<\mathrm{e}<0.172 \mathrm{~mm}$

Difference to $95 \%$ (with compensation) : $-0.004 \mathrm{~mm}<\mathrm{e}<0.046 \mathrm{~mm}$

Figure - I2. Differences between the measured points and the theoretical plane (carbide tool).

The position defect after compensation strongly decreased compared to normal milling. We observed that the $95 \%$ difference between the measured points and the theoretical plane is really smaller when we compensate. $77 \%$ of the surface points after compensation respect an interval of $\pm 0.01 \mathrm{~mm}$, but the whole surface is tilted because of the tool deflection. It now seems difficult to improve the model without going over to 5 axes in order to compensate for the tool paths simultaneously by translation and rotation. Moreover, in this test, the transition zones have not been taken into account in the simulation. To improve this result, we will soon set up a 5 -axis compensation and will manage the transition calculation at the depth of cut changes. The integration of our algorithm in a CAM system requires a tool engagement map definition correlated with a part's feedrate map which takes into account the machine tool dynamic. These maps allow to estimate the surface deviation. In 3-axis milling, we define a mean profile to calculate tool paths and locally control the feedrate. In 5-axis, we define tool paths with positioning of two points of a tool axis on the mirror surface. A distortion of this surface can be used to modify the part feedrate when its distribution is not continuous.

\section{CONCLUSIONS AND PROSPECTS}

Machining tool path generation is rather difficult in many ways. Three great classes of problem appear : the tool optimal laying on the machined surface, the generation of a numerical control program taking into account the machine controller's real capacities and the generation of a numerical control program taking into account the distortion aspects generated by the cutting process. We have specifically worked on the latter. We have shown that the distortion aspect due to the mechanical stresses during machining is particularly disadvantageous. No current CAM system has integrated this type of problem. To contribute to such an integration, we set up and evaluate a fast step to qualify a couple tool - workpiece material in order to predict the distortions undergone by a part during its machining. The method is 
based on the machining of a test plane. This first stage allows accurate assessment of the defects generated by the cutting process and calculation of the compensation. Nevertheless, a certain number of defects related to the non-rigidity of the workpiece and its fixture, also related to vibrational phenomena and the shock effects on attack and on exit of the cutter remain to be taken into account. In order to improve the machined parts quality, we set up a 3-axis method compensation. Finally, we hope to be able to propose a 5 -axis compensation method based on the model that we have just set up.

\section{REFERENCES}

1. A. AFFOUARD. "Introduction de l'interpolation polynomiale dans le cadre de l'usinage à 5 axes par le flanc", DEA de production automatisée LURPA ENS CACHAN, 2001.

2. F. MONIES, J.M. REDONNET, W. RUBIO, P. LAGARRIGUE. "Improved Positioning of a Conical Mill for Machining Ruled Surfaces: Application to Turbine Blades", J. of Engineering Manufacture, Vol. 214, Nº 2000, pp. 625-634.

3. TÖNSHOFF. "Optimal Tool Positioning for Five-Axis Flank Milling of Arbitrary Shaped Surfaces", Production Engineering, Vol. 7, N¹, 2000.

4. F. ABRARI, M. A. ELBESTAWI. "Closed form formulation of cutting forces for ball and flat end mills", Int. J. of Machining Tool Design Research, Vol. 37.1, 1997, pp. 17-27.

5. W. A. KLINE, R. E DEVOR, J. R. LINDBERG. "The prediction of cutting forces in end milling with application to cornerong cuts", Int. J. of Machining Tool Design Research, Vol. 22.1, 1982, 7-2.

6. T. SEO. "Intégration des effets de déformation d'outil en génération de trajectoires d'usinage" Thèse de Doctorat, Ecole Centrale de Nantes, 1998.

7. R. E. DEVOR, W. A. KLINE, W. J. ZDEBLICK. "A Mechanistic Model for the Force System in End Milling with Application to Machining Airframe Structures", Proceedings of eighth NorthllAmerican Manufacturing Research Conference: USA, Vol. 8, 1980, pp. 297-303.

8. A. LARUE, B. ANSELMETTI, B. SOULIER. "Influence des déformations des fraises sur la qualité des surfaces usinées", 7ème Colloque PRIMECA sur la Conception Mécanique Intégrée, La Plagne, 2000, pp. 334-341.

9. J. Y. HASCOET, J. J. LEE, A. DUGAS. "Machining simulator with dynamic error analysis on free 5urface machining", third. Int. Conference on Integrated Design and Manufacturing in Mechanical Engineering, Montréal, Canada, 2000. 